ISSN: 0213-2052 - eISSN: 2530-4100

DOI: https://doi.org/10.14201/shha2020384977

\title{
CUM CURA EXPLORATIS (LIV. XXII, 12,2): INTELIGENCIA MILITAR EN ROMA DURANTE EL SIGLO III A. C. ${ }^{1}$
}

\section{Cum cura exploratis (LIV. XXII, 12,2): Military Intelligence in Rome during the Third Century B.C.}

\author{
Pau VALDÉS MATÍAS \\ Universitat de Barcelona \\ pauvm84@hotmail.com
}

Fecha de recepción: 4-2-2020; aceptación definitiva: 19-06-2020

ORCID: https://orcid.org/0000-0002-1808-8771

RESUMEN: Las derrotas sufridas en los años iniciales de la Segunda Guerra Púnica han llevado a considerar que Roma no contaba con una inteligencia militar suficientemente desarrollada. Sin embargo, una revisión de los autores clásicos pone de manifiesto los problemas que plantea esta concepción. Por medio de un análisis de las evidencias escritas proponemos una nueva interpretación de la inteligencia militar romana durante el siglo III a. C.

Palabras clave: Ejército romano-republicano; siglo III a. C.; inteligencia militar; Guerras Púnicas.

1. Quiero agradecer los valiosos comentarios y anotaciones realizados por el Dr. Toni Ñaco y la Dra. Jerónima Riutort, así como a los revisores. Todos ellos han mejorado de forma notable los argumentos aquí presentados. 
ABSTRACT: The defeats suffered in the initial years of the Second Punic War have led to consider that Rome did not have a sufficiently developed military intelligence. However, a review of the classic authors reveals the problems posed by this conception. Through an analysis of written evidence we propose a new interpretation of Roman military intelligence during the third century B.C.

Keywords: Roman Republican Army; Third Century BC; military intelligence; Punic Wars.

La Segunda Guerra Púnica tuvo un gran impacto en la sociedad romana que la consideró uno de los grandes momentos de su historia y un punto de inflexión en la conquista del Mediterráneo. Esta visión aún hoy perdura. Esta contienda se ha considerado como una bisagra entre la ciudad arcaica y la que gobernará el Mediterráneo. Uno de los ámbitos en los que más se ha enfatizado este cambio ha sido en el militar, especialmente a raíz de las victorias iniciales de los cartagineses.

Entre los años 218-216 a. C. Roma sufrió numerosas derrotas, como Trasimeno o Cannas. Con el fin de explicarlas, se han desarrollado numerosos estudios analizando las diferencias entre los ejércitos romanos y cartagineses para dilucidar la razón detrás de los éxitos púnicos. Uno de los aspectos en los que más hincapié se ha puesto es en la gestión de la inteligencia militar por parte de Aníbal, así como de su empleo de estratagemas y emboscadas.

Este aspecto ha sido abordado desde múltiples perspectivas, destacándose que esta obsesión por las emboscadas en los autores clásicos es una racionalización de las derrotas ${ }^{2}$ o un modo de exagerar los estereotipos sobre los cartagineses ${ }^{3}$. Numerosos trabajos se han centrado en la capacidad romana para gestionar la inteligencia militar, existiendo un

2. LeIgh 2004, 47. Östenberg analiza las causas que suelen atribuirse a la derrota en el mundo romano siendo el ansia de gloria de los generales, enemigos traicioneros y la propia geografía las más notorias: ÖstenBerg 2014, 25-62 y ÖstenBerg 2018, 240-261. Así, las referencias al carácter traicionero de los enemigos de Roma pueden encontrarse para los galos (Plb. III, 71, 2-5), los germanos (Tac. Ann. II, 5, 3) o los íberos (Liv. XXVIII, 32; para una ilustrativa comparativa con el ejército romano vid. QueSADA 2006, 245-263). La batalla de las Horcas Caudinas es paradigmática en la caracterización de la geografía como un elemento adverso a los ejércitos romanos: HoRsfall 1982, 45-52. La descripción de Livio de la campaña del año 187 a. C. contra los ligures recoge estos tópicos con el fin de enfatizar el desempeño romano. Así, el teatro de operaciones destaca por lo escarpado y angosto. Asimismo, el enemigo ataca de forma constante, pero sin plantear una batalla (Liv. XXXIX, 1, 4-8).

3. FRONDA 2011, 245-246. 
consenso sobre su inexperiencia ${ }^{4}$. No será hasta la contienda con Aníbal que tendrá lugar un cambio en el tratamiento de la inteligencia militar por parte de Roma. Si bien algunos autores han negado esta posibilidad ${ }^{5}$, sus propuestas no han tenido éxito.

Se han planteado diversas hipótesis para esta transformación. Por un lado, producto de un proceso de aprendizaje cuyo punto culminante se daría al final de la guerra de la mano de Publio Cornelio Escipión el Africano (Cos. 205 a. C., 194 a. C.) ${ }^{6}$. Por el otro lado, gracias a las prórrogas en el mando de los magistrados al permitirles mayor experiencia y comprensión de la inteligencia ${ }^{7}$. En todas estas teorías se ha apuntado a problemas dentro del ejército como causantes de los errores ${ }^{8}$ o a otros ámbitos?.

Estos planteamientos han focalizado su estudio en la Segunda Guerra Púnica, sin plantear la gestión de la inteligencia militar en el marco del conjunto del siglo III a. C. De este modo, visto en perspectiva, resulta paradójico que una carencia tan grande como la que se le atribuye al ejército romano le permitiese realizar campañas tan complejas como las que documentamos a lo largo del siglo.

Asimismo, tampoco se ha considerado el papel que desempeñó la logística en el empleo de la inteligencia militar por parte de Roma. La gestión de los abastecimientos fue tornándose más compleja de forma paralela a la expansión romana. De este modo, los generales tuvieron que hacer frente a demandas que requirieron de una correcta aplicación y comprensión de la inteligencia militar.

4. El concepto de inteligencia militar, en la actualidad, hace referencia a la recolección, análisis y difusión de información sobre las capacidades, así como intenciones de enemigos actuales o potenciales. A su vez, podemos distinguir entre inteligencia estratégica y táctica. La primera abarca una perspectiva global analizando aspectos tanto militares como económicos, sociales o políticos. La segunda, por el contrario, se centra en la información sobre las fuerzas enemigas en una zona concreta. Sobre una visión general del concepto para el mundo antiguo: RanKov 2015, 541.

5. Southern 2006, 225; Palao 2016, 123-157.

6. Austin y Rankov 1995, 10, 15; Sheldon 2005, 61; Liddell Hart 1925, 251-252; Brizzi 1982,; Françols 2006, 313-328.

7. FOURNIE 2009, 518.

8. Rawlings 2007, 57; Fournie 2009, 512; Feugère 1993, 105; Rawson 1971, 21.

9. Buono-Core 2002, 74; Sheldon 1987, 54; Brizzi 1982. 
1. La inteligencia militar antes de la Segunda Guerra Púnica:

Roma DURANTE El Siglo III A. C.

El punto de partida de nuestro trabajo hay que situarlo en Frontino. Al narrar una estratagema de Quinto Fabio Máximo Ruliano (cos. 322 a. C.) refiere como en aquel entonces, en el año 310 a C., Roma aún no contaba con astutos métodos de exploración (sagaciores explorandi) ${ }^{10}$. El autor no señala qué diferencias aprecia y, para aumentar la confusión, encontramos otras estratagemas de una cronología similar a la anterior en su obra ${ }^{11}$. Contraponiendo esta afirmación con Livio, las diferencias aún son más difíciles de discernir. Sin embargo, en última instancia Frontino ya destaca la existencia de un sistema de inteligencia a inicios del siglo III a. C. aunque sea rudimentaria.

Las campañas contra etruscos y samnitas narradas por Livio muestran a una Roma que despliega toda una serie de prácticas propias de un Estado que conoce la inteligencia militar. Así, los diferentes magistrados emplean traductores ${ }^{12}$, espías ${ }^{13}$ exploratores $^{14}$, medidas para confundir a los enemigos ${ }^{15}$ o recurren a desertores para rendir ciudades ${ }^{16}$. También hay ejemplos de errores y mala gestión de la inteligencia militar ${ }^{17}$.

Brizzi descarta su veracidad ${ }^{18}$, al encontrar repeticiones en ellas, pero otros autores sí que aceptan las narraciones de Livio $^{19}$. Es indudable que la obra del patavino presenta pasajes problemáticos ${ }^{20}$, producto de las fuentes empleadas ${ }^{21}$. Por esta razón, no es extraño encontrar diferentes versiones de un mismo hecho, como el resultado de una batalla ${ }^{22}$, o una cierta elaboración en las causas de la derrota, como en las Horcas Caudinas ${ }^{23}$. Aun así, no deja de ser interesante la crítica de Livio a la mala gestión de la inteligencia militar de los cónsules ${ }^{24}$.

10. Fron. Str. I, II, 2.

11. Fron. Str. II, V, 1.

12. Liv. $X, 4,7-10$.

13. Liv. X, 20, 7-9.

14. Liv. IX, 23, 3; Liv. X, 10, 3-4; Liv. X, 27, 4-6; Fron. Str. I, II, 7.

15. Liv. X, 14, 13-4; Liv. X, 41, 5-7.

16. Liv. IX, 24, 3-13.

17. Liv. IX, 2, 2-4.

18. BRIZZI 1982, 8.

19. Cornell 1995, 355-357; Harris 1971, 77.

20. OAKLey 2005, 76-77, 228-229.

21. Liv. VIII, 40, 3-6.

22. Libourel 1973, 71-78.

23. Horsfall 1982, 45-52.

24. Liv. IX, 5, 7. 
El escenario descrito para este periodo adquiere una mayor consistencia al complementarlo con la información que nos proporciona Polibio. El autor griego destaca de forma positiva la experiencia de Roma en la guerra a raíz de sus luchas contra samnitas y galos, al haberla entrenado en el arte de la guerra ${ }^{25}$. Dadas las críticas de Polibio hacia los que no gestionan de forma adecuada la inteligencia ${ }^{26}$, es verosímil asumir que una parte de los datos que nos suministra Livio sean reales. Asimismo, la existencia de estas prácticas parece confirmada al contraponerlas con las noticias que nos ofrece la guerra entre Roma y Pirro.

En el año 280 a. C. el rey del Epiro acudió a la llamada de ayuda de Tarento para proteger la ciudad de la expansión romana. El monarca se había formado en los conflictos entre los sucesores de Alejandro Magno teniendo un papel destacado y gozando de gran experiencia militar ${ }^{27}$, asî como dominio de las estratagemas y del subterfugio ${ }^{28}$. Esta valoración positiva ha pervivido y numerosos autores valoran de forma positiva sus conocimientos militares ${ }^{29}$, así como su empleo de estratagemas ${ }^{30}$. Si bien conviene tener presente que esta valoración se deriva de una evidencia problemática $^{31}$.

Frente a la experiencia del epirota, Roma no parece encontrarse en desventaja. Las menciones que tenemos en los autores clásicos destacan aspectos como su organización del campamento ${ }^{32}$, pero no parecen extenderse en aspectos relativos a la inteligencia militar. Al respecto, podemos mencionar la captura de un espía del rey epirota por el cónsul Publio Valerio Lavinio (cos. 280 a. C. $)^{33}$. La batalla de Heraclea es producto de una correcta interpretación de las capacidades del enemigo por parte de los romanos, forzando la contienda para evitar que Pirro reciba refuerzos $^{34}$. En una línea parecida, en Ausculum, los cónsules escogen un terreno desfavorable para el rey epirota ${ }^{35}$ o recolectan sus tropas en función de los cambios de Pirro $^{36}$.

25. Plb. I, 6, 6-7.

26. Plb. III, 48, 1-3; Plb. VIII, 36, 2-3.

27. Dion. Hal. Ant. Rom. XX, 10, 1-2.

28. Plut. Pyrrh. 26, 5-6; Dion. Hal. Ant. Rom. XIX, 11; XX, 12, 1; Plut. Pyrrh. 11, 9-10.

29. Forsythe 2005, 352; Scullard 1969, r 120; Cornell 1995, 364; Kent 2019.

30. WhEELER 1988, 14.

31. Caire 2009, 233-247; Corbier 2009, 221-231; Rodríguez Horrillo 2009, 145-161.

32. Plut. Pyrrh. 16, 6.

33. Dion. Hal. Ant. Rom. XIX, 11.

34. Plut. Pyrrh. 16, 9.

35. Plut. Pyrrh. 21, 7; Fron. Str. II, II, 1.

36. Fron. Str. II, III, 21. 
El otro conflicto de gran importancia para nuestro trabajo es la Primera Guerra Púnica. La contienda estuvo marcada por los golpes de mano $^{37}$, engaños en los asedios ${ }^{38}$ y estratagemas diversas ${ }^{39}$, llevadas a cabo tanto por romanos y cartagineses ${ }^{40}$ como por las ciudades griegas ${ }^{41}$.

Durante el asedio de Agrigento en el 262 a. C., Zonarás afirma que, en parte, las dudas sobre qué acción llevar a cabo durante el sitio eran producto del miedo a una emboscada romana ${ }^{42}$. En una línea muy parecida, Frontino atribuye la victoria al general romano por el empleo de una estratagema ${ }^{43}$. Dado que Polibio no hace referencia a estos hechos, Lazenby se decanta por la versión del autor de Megalopolis, si bien destaca que no existe modo de saber cuál de las diferentes versiones es cierta ${ }^{44}$.

En el marco de la invasión de Marco Atilio Régulo (cos. 267) en África encontramos otro ejemplo. Cuando los cartagineses acuden desde Adi sus generales sitúan sus tropas en una colina que resulta inadecuada para sus fuerzas, algo que, debido a su experiencia ${ }^{45}$, los jefes romanos aprovechan en su beneficio para obtener una victoria sobre las fuerzas púnicas ${ }^{46}$. El hecho de que esta decisión táctica pudiese ser deliberada por parte de los cartagineses, con la finalidad de evitar la infantería romana en territorio llano, refuerza la capacidad del general romano ${ }^{47}$.

Finalmente, cabe destacar la estratagema desarrollada por Lucio Cecilio Metelo (cos. 251 a. C., 247 a. C.), que fue capaz de analizar la posición cartaginesa, así como el exceso de confianza del general púnico Asdrúbal, para atraerlo a un terreno escogido por él y preparado para neutralizar a sus elefantes ${ }^{48}$.

La afirmación más rotunda sobre la capacidad militar romana se produce en la narración de las campañas en los alrededores de Érice entre Amílcar y los romanos. En ella se destaca de forma positiva la gran cantidad de estratagemas y tácticas desplegadas, resaltando la gran variedad empleada por ambos bandos ${ }^{49}$.

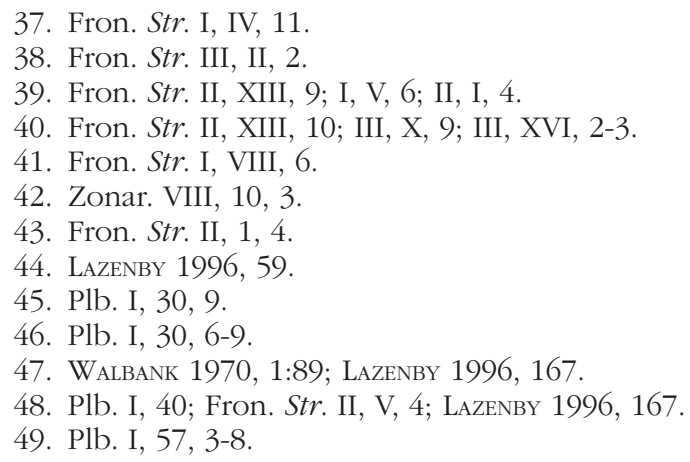


La valoración de la capacidad militar de Roma en la Primera Guerra Púnica es dispar. Vacanti destaca la experiencia con la que contaba Cartago en la gestión de la inteligencia ${ }^{50}$. Loreto valora, desde una perspectiva de la gestión de la contrainteligencia y a nivel macroestratégico, de forma muy negativa la capacidad de gestionar la inteligencia para discernir la Grand Strategy púnica por parte de Roma ${ }^{51}$, si bien no niega momentos de buena gestión en episodios concretos. En claro contraste, Lazenby no aprecia grandes diferencias entre los generales romanos y cartagineses en términos de competencia ${ }^{52}$. La excepción la constituye Amílcar Barca, cuya habilidad es destacada por los autores clásicos ${ }^{53}$ y por los autores modernos $^{54}$, con alguna excepción ${ }^{55}$.

En los años anteriores a la Segunda Guerra Púnica existen diferentes menciones importantes. Las campañas de Lucio Cornelio Escipión (cos. 259 a. C.) en el 259 a. C. en Cerdeña muestran diversos ataques nocturnos, así como fingir retiradas para tomar diversas poblaciones ${ }^{56}$. En el marco de las campañas contra los galos, Cayo Atilio Régulo (cos. 225 a. C.) capturó a forrajeadores enemigos, mediante tropas ejerciendo labores de reconocimiento, obteniendo datos sobre la posición gala ${ }^{57}$ y en base a ellos ocupó una posición. Por medio de la información con la que contaba, Lucio Emilio (cos. 225 a. C.) dedujo el inicio de los enfrentamientos entre las tropas de Cayo Atilio y los galos y acudió en su ayuda ${ }^{58}$. En la campaña de Acerra, los cónsules ocuparon los lugares estratégicos alrededor de la localidad, no permitiendo que esta recibiese ayuda ${ }^{59}$. En el decurso de la Segunda Guerra Ilírica encontramos el empleo de una estratagema por parte del cónsul Lucio Emilio Paulo (cos. 219 a. C.) para tomar la ciudad de Faros $^{60}$

Wheeler plantea la hipótesis de que el cognomen Sapiens está asociado a la habilidad militar y el empleo de estratagemas ${ }^{61}$. Si bien no

50. VACANTI 2012, 144-148.

51. LORETO 2007, 123-135, esp. 134-135.

52. Lazenby 1996, 166-167.

53. Diodoro Sículo, XXIII, 22.

54. Le Bohec 1996, 98-99; Brisson 1973, 90-91; Bagnall 2002, 44; Scullard 1969, 120; De Sanctis 1916, vol. III, 397; Goldsworthy 2002, 35-38; Quesada Sanz 2005, 138.

55. Hoyos 2015, 70; Hoyos 2003,

56. Fron. Str. III, IX, 4; X, 2.

57. Plb. II, 26. Walbank 1970, 1:205.

58. Plb. II, 27-28.

59. Plb. II, 34, 3-7.

60. Plb. III, 18, 9-19, 7

61. WHEELER 1998, 166-195. 
existen evidencias que puedan negar esta asociación de forma clara ${ }^{62}$, tampoco contamos con evidencias que nos permitan afirmar con seguridad esta relación. Sin embargo, diversos autores no descartan esta posibilidad ${ }^{63}$.

El conjunto de evidencias que hemos presentado nos ofrecen una imagen compleja de la gestión de la inteligencia militar romana durante el siglo III a. C. Contamos con una gran cantidad de menciones a su empleo, pero, a su vez, la validez de estas afirmaciones es cuestionada. Al mismo tiempo, diversos autores clásicos nos fijan una cierta periodización respecto a la capacidad romana al respecto.

Es evidente que a finales del siglo iv a. C. Roma contaba con medios para obtener inteligencia militar. Frontino destaca lo poco desarrollados que están, no su inexistencia. Por consiguiente, diversas de las menciones que hace Livio no pueden ser descartadas. Los aspectos vinculados a la inteligencia militar son coherentes con el relato presentado y existe poca duda sobre su veracidad ${ }^{64}$. Encontramos aciertos y éxitos importantes, como puede ser la estratagema de Lucio Postumio Megelo (cos. 305 a. C.) en el 305 a. C. ${ }^{65}$, pero también acciones o errores de envergadura ${ }^{66}$, así como éxitos fortuitos ${ }^{67}$. Asimismo, el empleo de exploradores es uno de los elementos que no solo va asociado a la gestión de la inteligencia militar. Estos jugaban un rol vital en la logística de un ejército. Por consiguiente, esta mezcolanza de resultados es, en gran medida, la que cabe esperar para un ejército gestionando inteligencia en el decurso de una campaña. Otros casos, como el de los espías, son más dudosos.

De este modo, la caracterización que nos ofrece Livio de la inteligencia militar se corresponde de forma acertada con la afirmación de Frontino. Sin embargo, en el periodo que abarca desde el 310 a. C. hasta la afirmación de Polibio $^{68}$ no apreciamos ninguna variación sustancial en los medios con los que se obtiene la inteligencia militar. Pese a este silencio por parte de los autores clásicos creemos que parte de esta evolución puede explicarse desde una perspectiva logística.

Rosenstein ha destacado como a partir del siglo iv a. C. el ejército romano tuvo que llevar a cabo campañas cada vez más lejanas y de mayor duración. Este hecho llevó a la introducción de los abastecimientos por

62. PoccetTi 2002, 698-699.

63. McDonnell 2006, 34; Pasco-Pranger 2012, 8.

64. LibOUREL 1973, 77.

65. Liv. IX, 44, 7-12.

66. Liv. IX, 38, 3.

67. Liv. IX, 31, 7-8.

68. Plb. I, 6, 6-7. 
parte del Estado, lo que permitía una mayor flexibilidad táctica. Así, podía atacar las infraestructuras enemigas, llevar a cabo asedios de forma más fiable y no estar limitada en el tiempo ${ }^{69}$. Sin embargo, Rosenstein se equivoca al afirmar que los ejércitos ya no dependían de abastecerse sobre el terreno ${ }^{70}$. La realidad es que existían toda una serie de recursos cuyo transporte planteaba demasiados problemas. Por consiguiente, elementos como el agua, forraje o madera fueron obtenidos sobre el terreno tanto en época republicana como imperial ${ }^{71}$.

Los condicionantes que implicaban estos recursos exigían toda una serie de medidas, así como un flujo de información constante. Este hecho, unido con la mayor presencia de las tropas en territorio enemigo, debió significar un mayor empleo y gestión de la inteligencia militar por parte de los ejércitos romanos. Más que un cambio en el modo como se obtenía la información, nos encontramos ante una mejor comprensión y empleo de esta. De este modo, también se explicaría la ausencia de cambios notorios en los métodos para obtener inteligencia en los autores clásicos.

En última instancia, el mejor testimonio de esta evolución en la gestión de la inteligencia militar son las campañas que desempeñó Roma contra dos potencias militares como Epiro y Cartago durante el siglo III a. C., en donde no encontramos menciones a una mala gestión de la inteligencia de forma sistemática.

\section{Inteligencia militar en la Segunda Guerra Púnica: una nueva interpretación}

Nuestra revisión de los conflictos del siglo III a. C. ha puesto de manifiesto la existencia de numerosas referencias al empleo de la inteligencia militar por parte de los ejércitos romanos. Sin embargo, estas evidencias por sí mismas no son excluyentes con los planteamientos que proponen una reforma de la inteligencia militar a raíz de las derrotas frente al general púnico.

\subsection{Inteligencia militar durante la Segunda Guerra Púnica}

Iniciaremos este apartado revisando las evidencias al empleo de Inteligencia militar por parte de los comandantes romanos en los años iniciales del conflicto hasta la batalla de Cannas. Esta periodización responde

69. Rosenstein 2004, 29-30.

70. Ibid., 30.

71. Valdés Matías 2017, 185-188, 198-225. 
al peso que tuvo esta derrota en la estrategia de ambas potencias, provocando diversos cambios ${ }^{72}$. Una transformación que también afectó la logística ${ }^{73}$, pero no se ha analizado desde la perspectiva de la inteligencia militar.

En las etapas iniciales contamos con menciones al empleo de tropas romanas en labores de exploración. Al desembarcar en Marsella Publio Cornelio Escipión (cos. 218 a. C.) envía un cuerpo de 300 jinetes junto con guías marselleses y galos con la finalidad de localizar a Aníbal y decidir en qué punto hacerle frente ${ }^{74}$. Una vez ya cruzados los Alpes, en las inmediaciones del Po, tanto Aníbal como Escipión reciben noticias de las cercanías de ambas fuerzas entre sí por medio de los forrajeadores ${ }^{75}$. Este hecho es el que lleva a ambos generales a enviar tropas para reconocer/

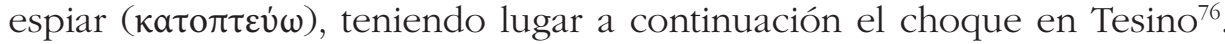
Es interesante resaltar que, en los prolegómenos de la batalla de Trebia, al analizar la situación de Aníbal, Livio destaca la existencia de espías galos (speculatoresque Galli) ${ }^{77}$ en ambos campamentos ${ }^{78}$.

Es en el tratamiento de la figura de Quinto Fabio Máximo (cos. 223 a. C.) donde encontramos más referencias a la gestión de la inteligencia. Después de recibir el ejército del legado Fulvio Flaco emprende la marcha hacia Aníbal, explorando con atención (cum cura exploratis) ${ }^{79}$. Unas labores de exploración que se repiten en diversas ocasiones, si bien en ocasiones con resultados adversos ${ }^{80}$.

El empleo de los bueyes por parte de Aníbal durante la emboscada en la zona de Falerno se ha convertido en una de las estratagemas por antonomasia del general púnico ${ }^{81}$. Sin embargo, conviene resaltar toda la labor de análisis y estudio de la geografía que conllevó la disposición de los diferentes efectivos para bloquear el avance púnico ${ }^{82}$. Si bien es una acción fallida, indica un análisis, estudio y comprensión de las rutas

72. Fronda 2010, 243.

73. ERDKAMP 1998, 166.

74. Liv. XXI, 26, 3-5; Plb. III, 41, 9.

75. Plb. III, 65, 3-4.

76. Plb. III, 65, 4-5.

77. Si bien es un término problemático, vid. Austin y Rankov, 1995, 54, su presencia encaja con la política de los galos durante estas etapas iniciales del conflicto.

78. Liv. XXI, 53, 11.

79. Liv. XXII, 12, 2.

80. Liv. XXII, $15,4-5$.

81. Polyaen. VI, 38, 8; Fron. Str. I, V, 28; Liv. XII, 15-18; Plb. III, 92, 8-94, 10. Scullard 1969, 193.

82. Liv. XXII, 15, 11. 
y puntos por los que se podía desplazar el ejército púnico ${ }^{83}$. En última instancia, la estratagema es recordada porque permitió al general púnico escapar de una trampa que no había sido capaz de prever. Quizá el mejor testimonio de importancia y habilidad que entrañaba esta acción queda reflejado en que es uno de los logros que los Elogia del foro de Augusto le atribuye a Fabio ${ }^{84}$.

También cabe resaltar que Livio, al analizar las perspectivas de Aníbal ante la división de los ejércitos de Fabio Máximo y Marco Minucio Rufo (cos. 221) en el año 217 a. C., se refiere al primero con el adjetivo sollertia. La vinculación de este término con el empleo de estratagemas es induda$b^{85}{ }^{85}$. En el caso del segundo, pese a las críticas que recibe ${ }^{86}$, hay que destacar su ataque a los forrajeadores de Aníbal en Geronio. En esta acción Minucio escoge el momento adecuado del día en que la dispersión de las tropas cartaginesas es mayor. Asimismo, también dispone sus fuerzas de modo que le permiten constreñir el movimiento de Aníbal y su capacidad de respuesta ${ }^{87}$.

Las campañas de Marco Atilio Régulo (cos. 227 a. C.) y Cneo Gémino Servilio (cos. 217) en el 216 a. C. contra Aníbal muestran los mismos patrones que las de Fabio Máximo, centrándose en hostigar al ejército de Aníbal en los momentos de debilidad y cuando está disperso ${ }^{88}$. El éxito atribuido por Livio a estas operaciones ha sido objeto de valoraciones dispares, Erdkamp lo considera exagerado ${ }^{89}$ mientras que Hoyos cree que la situación fue tan complicada como es descrita ${ }^{90}$. La escena que describe Polibio es más compleja ${ }^{91}$. El autor griego enfatiza el carácter de escaramuzas y acciones de poca envergadura, pero sí que conviene tener presente que no menciona ninguna celada o error en la inteligencia por parte romana ${ }^{92}$.

También en los prolegómenos de la batalla de Cannas encontramos numerosas menciones y referencias a las labores de exploración así como su importancia. Así, en los enfrentamientos previos entre Lucio Emilio Paulo (cos. 219) y Cayo Terencio Varrón (cos. 216), se hace explícita la importancia que tiene la exploración en los enfrentamientos ${ }^{93}$.

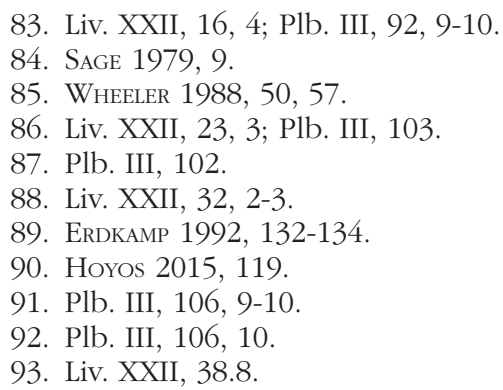


Las menciones de mayor interés son aquellas en las que aparece el prefecto Mario Estatilio. Este parece estar al mando de las labores de exploraciones. En dos ocasiones es mencionado realizándolas, ambas con éxito. En el primer caso, mediante la exploración cuidadosa (speculatusque omnia cum cura) consiguió ubicar la disposición del campamento cartaginés y advirtió la trampa preparada por Aníbal94. En el segundo caso, localiza a la columna cartaginesa tras descartar que la disposición en el campamento púnico fuese una trampa ${ }^{95}$. Es interesante resaltar que Livio menciona que para divisar a las tropas cartaginesas tuvo que ir más allá del campamento púnico y cruzar las montañas en las cercanías, ilustrando el alcance de las labores de reconocimiento ${ }^{96}$.

Los teatros de operaciones externos a la península itálica no han gozado de un peso importante en el análisis de la inteligencia romana. Sin embargo, nos permiten evaluar el ejército romano en territorios con grandes exigencias en este ámbito. Este rol se explica, en parte, por las evidencias dispares con las que contamos. En el caso de Sicilia, apenas las encontramos mientras que son mayores en la península ibérica. Así, del primer caso, documentamos una mención en los compases iniciales de la contienda. El pretor Marco Emilio, a raíz de la inteligencia militar obtenida por Hierón II, envía vigías a las atalayas situadas en la costa con el fin de controlar los movimientos de la flota púnica. Este hecho es el que permite divisar la flota púnica durante su aproximación nocturna ${ }^{97}$.

Por el contrario, las campañas de Cneo Cornelio Escipión (cos. 222) y Publio Cornelio Escipión (cos. 218 a. C.) nos proporcionan mucha más información, si bien algunas de las campañas presentan problemas ${ }^{98}$.

En el año 218 a. C. Cneo Cornelio Escipión (cos. 222 a. C.) embosca (insidia) a los lacetanos que acudían en defensa de los ausetanos durante la noche ${ }^{99}$, si bien Polibio no refiere este pasaje ${ }^{100}$. Este hecho ha generado diversas interpretaciones. Algunos autores han resaltado que esta ausencia es producto de la brevedad con la que el autor griego narra los hechos

94. Liv. XXII, 42, 4-5.

95. Liv. XXII, 43, 7.

96. Livio no especifica la distancia recorrida. Basándonos en las estimaciones de Ezov, Ezov 1996, 73-74, que toma como referencia evidencias más modernas, la distancia podría ser de unos quince kilómetros a medio día de distancia.

97. Liv. XXI, 49, 8.

98. Hoyos 2001, 68-92.

99. Liv. XXI, 61, 8.

100. Plb. III, 76, 12-3. 
de esa campaña ${ }^{101}$. Hoyos ha argumentado, desde nuestra perspectiva con acierto, a favor de su verosimilitud ${ }^{102}$.

La habilidad de Cneo también es valorada por Frontino al referir una victoria cerca de Indibile gracias a su capacidad para observar la moral y disposición de las tropas cartaginesas y sacar partido ${ }^{103}$. Al año siguiente, en el 217 a. C., reorganiza su plan en base a las noticias que recibe sobre el número de efectivos del ejército de Asdrúbal descartando una acción terrestre ${ }^{104}$. En esta misma campaña, es el empleo de dos naves marsellesas de exploración lo que le permite sorprender a la flota púnica ${ }^{105}$. Si bien Livio maximiza el efecto del pánico en la escuadra cartaginesa, Polibio destaca el éxito de la acción. Sósilo también destaca el éxito romano, aunque enfatiza mucho más el papel jugado por las naves masaliotas ${ }^{106}$.

A finales de este mismo año, Polibio relata cómo los dos Escipiones consiguieron obtener diversos de los rehenes retenidos en Sagunto mediante la ayuda de un íbero, Abelux, que engañó al jefe de la guarnición cartaginesa ${ }^{107}$. En su narración, Livio emplea el término sollertia para definir su plan ${ }^{108}$, un vocablo asociado a las estratagemas ${ }^{109}$. Si bien estamos de acuerdo en que el impacto de la acción es exagerado por los autores clásicos ${ }^{110}$, no creemos que nos encontremos ante una duplicación de la liberación de los rehenes de Cartago Nova por parte de Publio Cornelio Escipión el Africano (cos. 205 a. C.). Al respecto, coincidimos tanto con Hoyos como con Eckstein al destacar la veracidad de la acción ${ }^{111}$.

Finalmente, los movimientos previos a la batalla de Hibera son otro ejemplo. Livio indica que los Escipiones conocen el contenido de las cartas que Asdrúbal envía a Cartago, así como la marcha del cartaginés hacía la península itálica ${ }^{112}$. Una vez cruzado el Ebro, evalúan la situación y deciden iniciar el asedio de la ciudad de Hibera con la finalidad de hacer desistir al cartaginés de marchar a reunirse con su hermano. Esta acción denota que los Escipiones toman este curso de acción antes de

101. PÉREZ 1999, 29-30.

102. Hoyos 2001, 69-71.

103. Fron. Str. II, III, 1.

104. Plb. III, 95, 4-5.

105. Liv. XXII, 19-20, 2.

106. FGrH 176 F 1 . SChePENS 2013, 407.

107. Plb. III, 98-99; Liv. XXII, 22, 6-21.

108. Liv. XXII, 22, 6.

109. WHEELER 1988, 50.

110. Walbank 1970, 1:432.

111. ECKSTEIN 1987, 200; Hoyos 2001, 74.

112. Liv. XXIII, 28, 7. 
que Asdrúbal se encuentre en las inmediaciones y como producto de un análisis deliberado ${ }^{113}$.

\subsection{Inteligencia militar durante la Segunda Guerra Púnica: ¿una progresiva mejora?}

El conjunto de datos con los que contamos para los años previos a Cannas muestran una gestión constante por parte de las tropas romanas, tanto en la península ibérica como fuera de ella. Las referencias a la presencia de exploradores son continuadas. Su rol es tan importante que gozan de un papel importante en el debate entre Lucio Emilio Paulo y Cayo Terencio Varrón en los prolegómenos a la campaña de Cannas. También son mencionados en otros teatros de operaciones. Finalmente, hay que destacar que encontramos referencias al empleo de emboscadas por parte de las tropas romanas. Si bien este es un panorama rico en evidencias, estas, en última instancia, no difieren de las que hemos recabado previas a la guerra contra Aníbal. Por consiguiente, se podría pensar que la posibilidad de un cambio a raíz de las derrotas de esos años aún es verosímil.

Sin embargo, conciliar esta noción con los planteamientos de la obra de Polibio resulta difícil. En su libro VI, alaba la capacidad militar de Roma ${ }^{114}$, algo que resulta chocante si esta carecía de un sistema de inteligencia militar desarrollado, especialmente cuando es muy crítico con aquellos que no la emplean de forma adecuada ${ }^{115}$. Resulta una paradoja que, pese a contar con un sistema rudimentario, considerase a Roma como una experta en la guerra y la preparación de su ejército como una de las causas de su éxito ${ }^{116}$. No solo eso, al analizar las derrotas de Roma contra Aníbal no arguye en ningún momento una preparación inferior ${ }^{117}$.

No es la única contradicción que plantea esta hipótesis respecto a la obra de Polibio. En su análisis sobre el Estado romano, llama la atención sobre la gran capacidad de este para aprender y mejorar ${ }^{118}$. Así, resalta en diversas ocasiones esta cualidad y la enfatiza en la Primera Guerra Púnica ${ }^{119}$. De hecho, lo considera uno de los factores claves en su éxito.

113. Liv. XXIII, 28, 11-2.

114. Plb. I, 6, 6-7.

115. Plb. III, 48, 1-3; Plb. VIII, 36, 2-3.

116. Miltsios 2013, 17, 35-36; Champion 2011, 106-107.

117. Plb. XVIII, 28, 6. Champion 2011, 106-107.

118. WALKER 2017, 2.

119. Mitssios 2013, 39. Steinby considera que Polibio exagera esta inexperiencia por parte de Roma, Steinby 2014, 54-55, pero su valoración no es aceptada por todos, Rankov 2011, 152-153. 
Así, la relación entre Quinto Fabio Máximo y Marco Minucio Rufo se articula como un ejemplo de la capacidad de Roma para aprender y progresar a través de la experiencia durante los momentos más críticos de la Segunda Guerra Púnica ${ }^{120}$.

Así mismo, conciliar un aprendizaje derivado de Aníbal también resulta difícil. Esta idea es más compleja en Livio pues subyace una caracterización de Cartago como una Roma alternativa. Por esta razón, los cartagineses exhiben suficientes elementos positivos como para poder ser imitados, pero, a su vez, también cuentan con otros a rechazar ${ }^{121}$. De este modo, Los enfrentamientos con Aníbal sirven para definir qué aspectos deben adoptar los romanos para vencerle ${ }^{122}$. Tanto el general púnico como su ejército muestran virtus, pero su ejemplo es ambivalente pues también entraña un riesgo ${ }^{123}$. De todos los generales romanos, Escipión el Africano será el que estará más cerca de él pues su vida se constituye como un reflejo de la del púnico, en una suerte de Vidas Paralelas entre ambos $^{124}$, pero teniendo éxito donde él fracasó ${ }^{125}$.

Estas caracterizaciones de Livio también resuenan en la representación de los cartagineses como expertos en celadas producto de su perfidia. Algunos autores han considerado que la obsesión por las emboscadas en los autores escritos? es producto de una racionalización de las derro$\operatorname{tas}^{126}$ o un modo de exagerar los estereotipos sobre los cartagineses por parte romana ${ }^{127}$. Sin embargo, recientes estudios han llamado la atención a la complejidad de la representación de los cartagineses en este período ${ }^{128}$. Es más, White ha resaltado y recopilado numerosas evidencias de marchas nocturnas, cambio de posición o de construcción de campamentos tanto por parte púnica como romana ${ }^{129}$. Todas ellas son acciones que requieren gestionar la inteligencia. Por consiguiente, todas aceptadas dentro de la mentalidad romana ${ }^{130}$.

De hecho, pese a las similitudes que se trazan entre Escipión y Aníbal, a los ojos de Livio el primero en adoptar las prácticas púnicas es Fabio

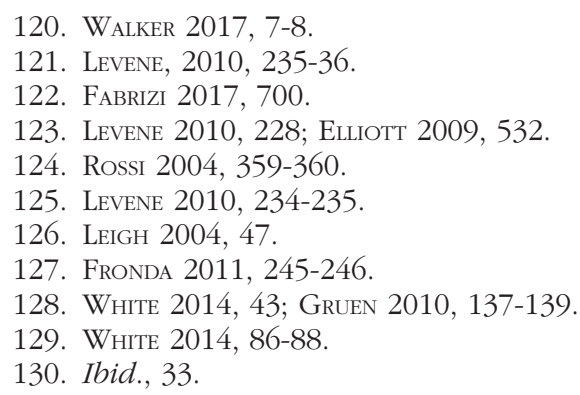


Máximo $^{131}$. La estrategia del dictador va asociada a acciones indirectas, desgastando al enemigo para librar batalla en unas condiciones escogidas por él ${ }^{132}$. La similitud con las acciones de Aníbal es resaltada por el patavino $^{133}$. Este hecho se llega incluso a representar a la inversa, con el general púnico empleando tácticas de Fabio ${ }^{134}$.

Por consiguiente, resulta muy difícil reconciliar una evolución de la inteligencia romana con estas evidencias pues implicaría que el autor griego llevó a cabo un análisis totalmente erróneo tanto de las capacidades militares de Roma como de los éxitos de Aníbal. Asimismo, esta evolución constituiría un claro ejemplo de una de las causas que, para Polibio, explican el éxito romano y, por consiguiente, resultaría extraño que fuese omitida en su análisis.

Por el contrario, si entendemos este aprendizaje como una consecuencia de las prácticas cartaginesas, las evidencias al respecto no son suficientes ni concluyentes. En primer lugar, porque se trata de una caracterización deliberada por parte de autores como Livio. En segundo lugar, ya existen toda una serie de prácticas que encajan dentro del ámbito de la inteligencia militar que son llevadas a cabo por parte de Roma. Finalmente, la ausencia de esta adaptación en Polibio sería de nuevo una sorpresa. Especialmente cuando ya ha representado en otras situaciones esta capacidad de Roma para adaptar prácticas y elementos de sus rivales, como la evolución de las naves durante la Primera Guerra Púnica ${ }^{135}$.

Un análisis en detalle del conjunto del conflicto parece poner de manifiesto esta progresión en la gestión de la inteligencia por parte de Roma, más bien se aprecian unas fluctuaciones considerables. Los dos primeros años del conflicto están marcados por los fracasos de Roma en este campo. En el año 218 a. C., tienen lugar emboscadas en Trebia ${ }^{136}$, en las cercanías de Tarraco $^{137}$ y en Liguria ${ }^{138}$. Un número parecido al que se registran al año siguiente, como las que tienen lugar en Trasimeno ${ }^{139}$, contra Marco Minucio Rufo ${ }^{140}$ o África ${ }^{141}$. Sin embargo, también encontramos éxitos por parte romana. De hecho, en el año 218 a. C. registramos hasta cuatro

131. Levene 2010, 229-230; Elliott 2009, 533.

132. Bagnall 1999, 184-185.

133. Liv. XXII, 16, 5.

134. Levene 2010, 230, nota 186; Fabrizi 2017, 692.

135. Plb. I, 61, 3.

136. Plb. III, 71-4; Liv. XXII, 54.

137. Plb. III, 76, 9-10; Liv. XXI, 61, 2.

138. Liv. XXI, 59, 10.

139. Plb. III, 84-5; Liv. XXII, 4, 4; Plb. III, 86, 3-5; Liv. XXII; 8, 1.

140. Plb. III, 104-5, 6; Liv. XXII, 28, 3-14.

141. Liv. XXII, 31, 1-5. 
acciones culminadas satisfactoriamente por parte de Roma ${ }^{142}$. Un número elevado, pues en solo cuatro años más atestiguamos un número de éxitos superior. Por su parte, para el año 217 a. C. documentamos tres éxitos ${ }^{143}$.

Entre los años 216 al 213 se constata un descenso en los fracasos por parte de Roma en la gestión de la inteligencia. A su vez, esta dinámica coincide con un incremento en los éxitos. De hecho, los años 215 a. C. ${ }^{144}$ y 214 a. C. ${ }^{145}$ son especialmente fructíferos para la inteligencia romana, con diversos éxitos.

Esta tendencia se rompe en el año 212 a. C. ${ }^{146}$, que constituye el tercer año con mayor fracaso en la inteligencia militar de todo el conflicto, solo por detrás de los dos años iniciales. A partir de este momento, se produce un descenso de los fracasos. Si bien siguen estando presentes, como en los años 208 a. C. ${ }^{147}$ y 206 a. C. ${ }^{148}$. Por el contrario, los años 210 a. C. ${ }^{149}$ y 207 a. C. ${ }^{150}$ son de los más exitosos para Roma, igualando al año 214 a. C., pero aún por debajo de 215 a. C.

En el año 218 a. C. las diferentes emboscadas registradas tienen lugar en diferentes escenarios de guerra. Por el contrario, el grueso de las del año 217 a. C. sucede en la península itálica ${ }^{151}$. A partir de entonces apenas hay registro de acciones similares hasta el 214 a. C. cuando Hannón pone una celada a Tiberio Sempronio Graco (cos. 215 a. C.) en Lucania ${ }^{152}$. Otro dato interesante es la escasez de noticias sobre emboscadas que tienen lugar fuera de la península itálica. Hay menciones a las que tienen lugar en Liguria y la península ibérica en el 218 a. C. ${ }^{153}$. Otra en el 217 a. C. ${ }^{154}$ y, finalmente, la última referencia es del año 215 a. C. ${ }^{155}$

142. Liv. XXI, 46, 3-5; XXI, 46, 3-5; XXI, 49, 9-12; XXI, 61, 8.

143. Liv. XXII, 12, 2; XXII, 16, 1-5; XXII, 24, 6-10.

144. Liv. XXIII, 34, 3-9; XXIII, 34, 10-12; XXIII, 35, 10-16; XXIII, 41, 8-9; XXIII, 44, 4; XXIV, 3, 16-7.

145. Liv. XXIV, 14, 2; XXIV, 19, 1-3; XXIV, 36, 1; XXIV, 40, 8-16.

146. Plb. VIII, 28, 1-2; Liv. XXV, 9, 5-7; Liv. XXV, 15, 1-17; Plb. VIII, 36, 1-2; Liv. XXV, 16 , 7-25; XXV, 17, 1-3; Liv. XXV, 21.

147. Liv. XXVII, 26, 4-5; XXVII, 27; XXVII, 31, 2.

148. Liv. XXVIII, 11, 13-4; XXVIII, 46, 8.

149. Liv XXVI, 38, 11-14; XXVI, 39, 20-23; XXVI, 42, 5-6; XXVII, 5, 9-11. También podrían añadirse las campañas de los Escipiones en las inmediaciones de Castrum Album, pero su historicidad es muy dudosa.

150. Liv. XXVII, 36, 1-4 y XXVII, 39, 1-3; XXVII, 41, 6-42, 8; XXVII, 43, 8-10; XXVII, 47, 1-5; XXVIII, 1, 6-2, 2.

151. Plb. III, 84-5; Liv. XXII, 4, 4; Plb. III, 86, 3-5; Liv. XXII; 8, 1; Liv. XXII, 28, 5-29.

152. Liv. XXIV, 20, 1-2.

153. Plb. III, 76, 9-10; Liv. XXI, 61, 2; Liv. XXI, 59, 10

154. Liv. XXII, 31, 1-5.

155. Plb. III, 118, 6-7; Liv. XXIII, 24, 6. 
El tratamiento de la contienda por los autores clásicos se focaliza en los años iniciales en la figura de Aníbal y las grandes derrotas romanas. Por el contrario, a partir de la irrupción de Escipión, este pasa a ser el centro de las narraciones de los autores clásicos. De este modo, toda una serie de campañas que se desarrollan en la península itálica pasan a ser condensadas y reciben escasa mención ${ }^{156}$.

Este cambio el foco de la narración de los autores clásicos es importante si lo comparamos con la recopilación realizada por Louis Rawlings de los enfrentamientos en la península itálica en los que tomó parte Aníbal ${ }^{157}$. Se puede apreciar cierta correlación entre estos con la inteligencia militar romana. Así, vemos como durante los primeros años se registran el mayor número de enfrentamientos. A partir del 215 a. C. descienden hasta repuntar entre los años 212-210 a. C. y de nuevo descender paulatinamente hasta el último enfrentamiento en el año 203 a. C. Esta dinámica parece coincidir con la importancia que le otorga Livio a la caída de Salapia en manos romanas en el 210 a. C. ${ }^{158}$. Este hecho marca el fin de la superioridad en la caballería del general púnico. La importancia que tenía esta había sido destacada en numerosas ocasiones por parte de los autores clásicos ${ }^{159}$.

Este repaso realizado pone de manifiesto que es una actividad continuada con diversos repuntes en éxitos y fracasos, producto de dinámicas específicas a las campañas o teatros de operaciones. Por consiguiente, no parece que se pueda deducir una evolución en la gestión de la inteligencia militar.

\section{PROCESANDO LA INTELIGENCIA MILITAR: EXPERIENCIA}

Estos altibajos que hemos documentado podrían atribuirse a la experiencia de la persona en el mando. Así, los momentos en que documentamos un gran número de errores podrían deberse a la presencia de generales sin experiencia mientras que los periodos con éxitos fuesen producto del mando de gente experimentada. Este planteamiento ${ }^{160}$ tiene problemas a nivel metodológico.

156. Como por ejemplo: Liv. XXIX, 36; XXIX, 38.

157. Rawlings 2011, 301-302.

158. Liv. XXVI, 38, 14.

159. Plb. III, 101, 8-11.

160. FournIE 2009, 518. 
En primer lugar, se establece una relación directa entre el fracaso en la inteligencia militar con su desconocimiento o ausencia. Esta equiparación simplifica la complejidad a todo el proceso de gestión de la inteligencia militar. La crítica de Perley al trabajo de Austin y Rankov es pertinente, pues la propuesta de estos autores ha derivado en una caracterización de la inteligencia como "buena" o "mala" de forma simplista. De hecho, la inteligencia puede probarse como errónea y pese a ello ser buena en términos de cómo fue recogida y analizada ${ }^{161}$.

$\mathrm{Al}$ analizar los errores en la inteligencia hay que tener presente que estos eran habituales y que no se pueden evitar. Una idea que ya apunta Polibio $^{162}$ y que aún sigue vigente ${ }^{163}$. Asimismo, todo el proceso de gestión de la información es complejo y la toma de decisiones se produce en un contexto cambiante ${ }^{164}$. Por consiguiente, no es extraño que existan errores $^{165}$ pues, incluso cuando se toman precauciones ${ }^{166}$, diversos avatares pueden influir. Jenofonte, por ejemplo, advierte sobre la posibilidad que la información no llegue a tiempo ${ }^{167}$. Por lo tanto, no existe una relación directa entre los errores y la falta de experiencia o conocimientos. Al contrario, encontramos numerosos ejemplos de generales de demostrada veteranía fallando en este campo. El caso más paradigmático es César, muy bien valorado en la gestión de la inteligencia ${ }^{168}$, y su invasión a Britania $^{169}$, pero para nuestro periodo de estudio hay otros ejemplos reveladores.

Quizá el más ilustrativo corresponde a la decisión de Fabio Máximo, general de demostrada habilidad en la gestión de la inteligencia como hemos visto, de creer a los emisarios de Metaponto cuando en realidad era una trampa ${ }^{170}$. Otro caso tiene lugar en el año 200 a. C., al iniciarse la Segunda Guerra Macedónica, Publio Sulpicio Galba, cónsul del año, recibió la provincia de Macedonia. Este nombramiento era el punto culminante de una larga carrera política. En el año 211 a. C. había sido nombrado cónsul y había defendido la ciudad de Roma ante el avance de

161. PerLey 2016, 4.

162. Plb. VIII, 36, 2-3; IX, 12, 4-5.

163. Betts 1978, 61; Betts 2002, 53; Perley 2014, 165; Handel 1984, 229.

164. Guan Kwa 2017, 111.

165. BAUdET et al. 2017, 12.

166. Plb. VIII, 36, 2-3.

167. X. Eq. Mag. 4, $4-8$.

168. Austin y Rankov 1995, 10.

169. SHELDON 2002, 77-100.

170. Liv. XXVII, 16, 13-16. 
Aníbal ${ }^{171}$. En el 210 a. C. fue procónsul en Grecia' ${ }^{172}$ y se mantuvo en el cargo hasta el 205 a. C. que fue substituido ${ }^{173}$. Pese a esta larga experiencia militar, durante las campañas contra Filipo V sufre una emboscada durante las operaciones de forrajeo ${ }^{174}$, que le acarreó duras críticas ${ }^{175}$.

En segundo lugar, ha existido una cierta tendencia a menoscabar la preparación de los generales romanos. Una noción errónea tal como ha planteado Quesada ${ }^{176}$. Autores como Hoyos, pese a ser crítico con numerosos generales romanos, en última instancia considera que estos estuvieron por encima de la media cartaginesa ${ }^{177}$. Cuando un aristócrata romano llegaba al consulado contaba con experiencia durante su servicio como tribunus militum y, muy posiblemente, como legatus ${ }^{178}$, considerándose con la experiencia en numerosas campañas ${ }^{179}$. Durante la contienda, en diez de los dieciocho años al menos uno de los cónsules del año ya había ostentado algún cargo político de importancia. Hasta el año 207 a. C., con la victoria sobre Asdrúbal en Metauro, no se romperá esta tendencia.

En tercer lugar, se han asumido como habituales las prórrogas en el mando, así como una finalidad únicamente militar. Lazenby reduce su importancia ${ }^{180}$. Durante los años de la guerra no se constata un descenso en el acceso a la magistratura, repitiendo consulado solo cuatro magistrados. Asimismo, solamente en una ocasión dos cónsules habían ostentado antes el cargo $^{181}$. Incluso en el año 212 a. C. vemos como todos los comandantes en la península itálica son cambiados por completo ${ }^{182}$. De hecho, una de las conclusiones del análisis de Lazenby sobre la guerra es que los romanos consideraron su sistema de elección de generales de forma anual exitoso y lo prolongaron con posterioridad ${ }^{183}$.

De especial interés para nuestro trabajo revisten los sucesos de los años 208-7 a. C. En el 208 a. C. los cónsules Marcelo (cos. 222 a. C.) y L. Crispino (cos. 208 a. C.) murieron en sendas emboscadas. Ambos contaban con una extensa experiencia militar. Marcelo en aquel momento

171. Broughton y Patterson 1951, vol. 1, 272.

172. Ibid., 1:280.

173. Ibid., 1:303.

174. Liv. XXXI, 36, 9-11.

175. Liv. XXXI, 38, 1-2.

176. Quesada Sanz 2015, 70-71; Quesada Sanz 2017, 213.

177. Hoyos 2015, 223-225; Hoyos 2013, 729.

178. LazenBy $1978,6$.

179. Campbell 1982, 20; Harris 1979, 10-16.

180. Fournie 2009; Scullard 1969, 226.

181. Lazenby 1978, 6, 253.

182. Ibid., 6, 109.

183. Ibid., 248. 
ostentaba el consulado por quinta vez además de haber visto prorrogado su imperium en diversas ocasiones. En el marco de las elecciones del año 208 a. C. Livio inserta una amonestación del Senado a la conducta de ambos generales, enfatizando la necesidad de contar con comandantes cuya virtus fuese capaz de resistir la fraus punica ${ }^{184}$.

La elección del 207 a. C. también resulta interesante por la presencia de Claudio Nerón. Había servido como pretor en el asedio de Capua y posteriormente había sido enviado a Hispania. Las referencias a su experiencia en la obra de Livio no son brillantes ${ }^{185}$, una valoración que es compartida por Hoyos $^{186}$. Fournie, por otro lado, la interpreta más positivamente ${ }^{187}$.

Livio destaca que fracasó en la tarea encomendada por Marcelo en Nola, al no conseguir atacar la retaguardia de Aníbal, recibiendo una fuerte reprimenda del cónsul ${ }^{188}$. En la península ibérica sufrió la fraus punica de Asdrúbal en Lapides Atri ${ }^{189}$. Finalmente, Livio enfatiza su carácter impulsivo, calificándolo con el término promptiorem acrioremque. Este término puede denotar carácter energético o vigoroso, pero tal como lo emplea Livio parece más lógico decantarse por un carácter ansioso, denotando una acción apresurada, sin meditar ${ }^{190}$. De hecho, todo este proceso guarda muchas similitudes con el de los generales derrotados por Aníbal ${ }^{191}$. Por consiguiente, en un momento clave del conflicto, pues se esperaba la llegada de Asdrúbal a la península itálica ${ }^{192}$, Roma prefirió poner al frente de sus ejércitos a un general sin amplia experiencia en un momento crítico y con unos antecedentes que no hacían de él un experto para hacer frente a la fraus punica.

Otro pasaje de gran significación es cuando Fabio Máximo se opone al nombramiento de Otacilio (Pr. 217 a. C.) como cónsul del 214 a. C. ${ }^{193}$. Por medio del cunctator, Livio realiza un alegato a favor de priorizar en el mando a aquellos que cuentan con más experiencia militar ${ }^{194}$. Sin embargo, este pasaje presenta diversos problemas. Así, Fabio Máximo se

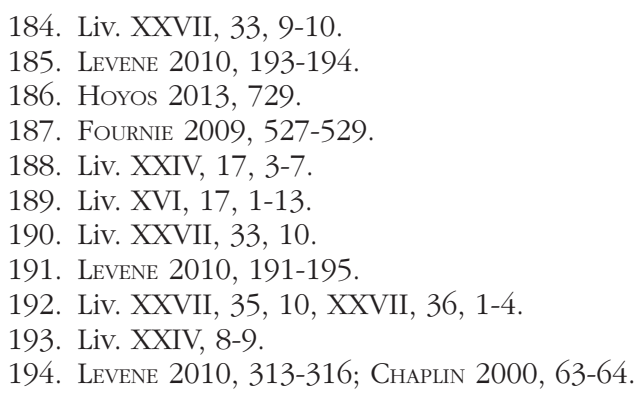


opone a la elección de Otacilio, pese a que este había desempeñado con eficacia su labor en la flota ${ }^{195}$. Sin embargo, si contraponemos la valoración de Livio con la que realiza de Escipión el Africano, queda patente que para él la línea divisoria entre un general exitoso y uno que no lo es resulta difícil de discernir y no se reduce a la experiencia militar ${ }^{196}$.

Finalmente, la experiencia en el mando no implica un aprecio de la inteligencia militar. Estudios sobre época moderna ponen de relevancia la tenue relación, así como el peso de otros factores en las decisiones, incluso por encima de la propia inteligencia.

El caso más llamativo se corresponde con los generales aliados durante la Segunda Guerra Mundial. Muchos de ellos no comprendieron el potencial de los recursos disponibles, como el sistema Ultra ${ }^{197}$. Generales con reconocido aprecio a la inteligencia la ignoraron en momentos determinados ${ }^{198}$. Asimismo, tras grandes éxitos en el campo de la inteligencia se cometieron errores básicos ${ }^{199}$. Incluso en la actualidad, donde la inteligencia militar goza de gran relevancia, los generales de carrera no parecen mostrar una mejor comprensión por la inteligencia. Paradójicamente, muchos de ellos expresan un desconocimiento sobre su gestión ${ }^{200}$. Por consiguiente, valorar la inteligencia y su empleo no guarda una relación directa con la experiencia en el mando, estando su uso condicionado por la formación del general ${ }^{201}$, la capacidad para interpretar los datos ${ }^{202} \mathrm{O}$ los imperativos políticos ${ }^{203}$.

El estudio de estos procesos no ha tenido un rol destacado dentro de los trabajos sobre la inteligencia militar romana. Sin embargo, conviene destacar que, para la actualidad, este es un campo de estudio aún incipiente ${ }^{204}$. Para el mundo antiguo, contamos con información muy poco detallada que no nos permite conocer los factores que condicionaron las decisiones. Quizá podemos atisbar parte de esta dinámica en los prolegómenos del choque del combate en Tesino. Tanto Escipión como Aníbal

195. Nombrado pretor al mando de la flota en Lilibeo en el 217 a. C. (Liv. XXII, 7, 7-8), fue prorrogado hasta el 215 a. C. (Liv. XXII, 37, 13; XXIII, 32, 20).

196. LEVENE 2010, 313-314.

197. Deutsch 1988, 242.

198. Ibid., 203-208.

199. Ibid., 240-253.

200. WolfBerg 2016, 1, 7.

201. Кам 2004, 87; Betтs 1978, 81.

202. SHELDON 2005, 287.

203. GENTRY 2008, 255.

204. Wolfberg 2016, 3 . 
DURANTE EL SIGLO III A. C.

muestran dudas sobre la información que reciben ${ }^{205}$, desdeñándola al principio y no es hasta el final que la aceptan.

\section{Conclusiones}

En base a Frontino y Polibio, entre los años finales del siglo iv a. C. y en los años previos al enfrentamiento contra Pirro, Roma mejoró su gestión de la inteligencia militar. Al respecto, la mayor distancia de las campañas militares así como las necesidades logísticas jugaron un papel importante. Durante este siglo las fuentes escritas ponen de manifiesto el empleo de diversos métodos para obtener inteligencia militar. Estos no parecen mostrar grandes diferencias respecto a los que pueden practicar los rivales de Roma. La existencia de un sistema de inteligencia militar es la única forma de explicar que, a lo largo del siglo III a. C., Roma fuese capaz de enfrentarse con garantías a generales expertos como Pirro o los diferentes mandos cartagineses durante la Primera Guerra Púnica.

Al iniciar la Segunda Guerra Púnica Roma estaba preparada para hacer frente a situaciones que requerían el empleo de la inteligencia militar. Los primeros años destacan por derrotas con errores de envergadura así como éxitos. Desde una perspectiva metodológica, el enorme peso atribuido a estos errores ha condicionado la lectura de los datos. Nuestra revisión ha tratado de poner de manifiesto que no parece atestiguarse un cambio en la inteligencia militar conforme el conflicto avanza, documentándose altibajos tanto para Roma como para Cartago.

Es importante destacar que estos errores se producen tanto por generales con poca experiencia como veteranos pues el tiempo en el mando no incide de forma relevante en los fracasos en la inteligencia militar o en un mayor aprecio por ella. Por consiguiente, no parece que podamos establecer una figura clave en la reforma de la inteligencia militar romana. De hecho, siguiendo las evidencias, más que Escipión el Africano, esta posición debería corresponder a Fabio Máximo.

Queda claro que los enfrentamientos contra Aníbal demostraron la genialidad militar del púnico, que fue capaz de superar en todos los campos a los generales romanos. Sin embargo, estas derrotas no marcaron ningún cambio. Los altibajos que muestran los autores clásicos son producto de las decisiones de los generales, así como su estrategia, tal como

205. Plb. III, 41, 5-9; III, 61, 1-7; Liv. XXI, 26, 4-5. 
puede apreciarse en el caso de Aníbal, y no derivados de cambios estructurales tanto en la gestión como en la consideración de la inteligencia militar.

\section{Bibliografía}

Austin, N. J. E. y Rankov, B. (1995): Exploratio. Military and Political Intelligence in the Roman World from the Second Punic War to the Battle of Adrianople, London and New York: Routledge.

Bagnall, N. (1999): The Punic Wars. Rome, Carthage and the struggle for the Mediterranean, London: Pimlico,

Bagnall, N. (2002): The Punic Wars 264-146 BC, Essential Histories, Oxford: Osprey Publishing.

Baudet, F., Braat, E., van Woensel, J. y Wever, A. (2017): «Military Intelligence: From Telling Truth to Power to Bewilderment?», en Baudet, F., Braat, E., van Woensel, J. y Wever, A. (eds.): Perspectives on Military Intelligence from the First World War to Mali. Between Learning and Law, Berlin: Springer, pp. 1-22.

BetTs, R. K. (1978): "Analysis, War, and Decision: Why Intelligence Failures Are inevitable», World Politics 31, n. ${ }^{\circ}$ 1, pp. 61-89.

BetTs, R. K. (2002): «Fixing Intelligence», Foreign Affairs 81, n. ${ }^{\circ} 1$, pp. 43-59.

Brisson, J.-P. (1973): Carthage ou Rome? Les grandes études historiques, Fayard.

BRIzzI, G. (1982): I sistemi informativi dei Romani. Principe e realtà nell'età delle conquiste oltremare (218-168 a.C.), Historia - Einzelschriften, 39, Wiesbaden: Frank Steiner.

Broughton, T. R. S. y Patterson, M. L. (1951): The Magistrates of the Roman Republic. Vol. I: 509 BC-100 BC, 2 vols., Philological Monographs, New York: American Philological Association.

Buono-Core, R. (2002): «Relaciones, información, espionaje y servicios de inteligencia en Roma», Valparaiso, XI, pp. 65-83.

CAIRE, E. (2009): «D'Héraclée à Ausculum: les traditions historiographiques", Pallas 79, pp. 233-247.

Campbell, B. (1982): "Teach yourself how to be a general", Journal of Roman Studies, 77, pp. 13-29.

Champion, C. B. (2011): «Polybius and the Punic Wars», en Hoyos, D. (ed.): A Companion to the Punic Wars, Blackwell Companions to the Ancient World, Malden: Blackwell Publishing, pp. 95-110.

Chaplin, J. D. (2000): Livy's Exemplary History, New York: Oxford University Press. 
Corbier, P. (2009): "Pyrrhus en Italie, réflexion sur les contradictions des sources", Pallas 79, pp. 221-231.

Cornell, T. J. (1995): The beginnings of Rome. Italy and Rome from the Bronze Age to the Punic Wars (c. 1000-264 BC), Routledge History of the Ancient World, London and New York: Routledge Press.

Deutsch, H. C. (1988): "Commanding generals and the uses of intelligence», Intelligence and National Security, 3, n. ${ }^{\circ}$ 3, pp. 194-260.

Eckstein, A. M. (1987): Senate and General. Individual decision-making and Roman foreign relations 264-194 B.C., Berkeley: University of California Press.

ElLiotт, J. (2009): "Ennius' "Cunctator" and the history of a gerund in the Roman historiographical tradition", The Classical Quarterly, 59, n. ${ }^{\circ}$, pp. 532-542.

ERdKamp, P. (1992): "Polybius, Livy and the Fabian Strategy», Ancient Society 23, pp. 127-147.

ERDKAMP, P. (1998): Hunger and the sword. Warfare and food supply in Roman republican wars (264-30 B.C.), Amsterdam: J.C. Gieben.

Ezov, A. (1996): "The "Missing Dimension" of C. Julius Caesar», Historia: Zeitschrift für Alte Geschichte, 45, n. ${ }^{\circ}$ 1, pp. 64-94.

FAbrizi, V. (2017): "The Cloud that (...) Sat on the Mountaintops": A Narratological Analysis of Space in Livy's Account of Quintus Fabius Maximus' Dictatorship", American Journal of Philology 138, n. ${ }^{\circ} 4$, pp. 673-706.

Feugère, M. (1993): Les armes des romains. De a République à l'Antiquité tardive, Collection des Hesperides, Éditions Errance.

Fonsythe, G. (2005): A Critical History of Early Rome. From Prebistory to the First Punic War, Berkeley-Los Angeles-London: University of California Press.

Fournie, D. A. (2009): «Harsh Lessons: Roman Intelligence in the Hannibalic War", International Journal of Intelligence and CounterIntelligence 17, n. ${ }^{\circ}$, pp. 502-538.

FrançoIs, Pl. (2006): "Externo more: Scipion l'Africain et l'hellénisation», Pallas, 70, pp. 313-328.

Fronda, M. P. (2010): Between Rome and Carthage. Southern Italy during the Second Punic War, Cambridge University Press.

Fronda, M. P. (2011): "Hannibal: Tactics, Strategy, and Geostrategy», en Hoyos, D. (ed.): A Companion to the Punic Wars, Blackwell Companions to the Ancient World, Malden: Blackwell Publishing, pp. 242-59.

Gentry, J. A. (2008): "Intelligence Failure Retrained», Political Science Quarterly 123, n. ${ }^{\circ}$ 2, pp. 247-270. 
Goldsworthy, A. (2002): Las Guerras Púnicas, Ariel Grandes Batallas, Barcelona: Ariel.

Guan Kwa, C. (2017): «Postmodern Intelligence: Strategic. Warning and Crisis Management, en Baudet, F., Braat, E., van Woensel, J. y Wever, A. (eds.): Perspectives on Military Intelligence from the First World War to Mali. Between Learning and Law, Berlin: Springer, pp. 97-118.

Handel, M. I. (1984): "Intelligence and the problem of strategic surprise», Journal of Strategic Studies 7, n. ${ }^{\circ}$ 3, pp. 229-281.

HaRRIS, W. V. (1971): Rome in Etruria and Umbria, Oxford: Claredon Press. HaRris, W. V. (1979): War and Imperialism in Republican Rome 327-70 BC, Oxford: Claredon Press.

Horsfall, N. (1982): «The Caudine Forks: Topography and Illusion», Papers of the British School at Rome, 50, pp. 45-52.

Hoyos, D. (2001): «Generals and Annalists: geographic and chronological obscurities in the Scipios' campaigns in Spain, 218-211 B.C.», Klio, 83, n. ${ }^{\circ}$ 1, pp. $68-92$.

Hoyos, D. (2003): Hannibal's Dynasty. Power and politics in the western Mediterranean, 247-183 BC, London: Routledge Press.

Hoyos, D. (2013): "The Second Punic War", en Campbell, B., Lawrence, A. T.: The Oxford Handbook of Warfare in the Classical World, New York: Oxford University Press, pp. 715-733.

Hoyos, D. (2015): Mastering the west. Rome and Carthage at War, Oxford: Oxford University Press.

Kaм, E. (2004): Surprise attack. The Victim's Perspective, CambridgeLondon: Harvard University Press.

Kent, P. A. (2019): A History of the Pyrrbic War, Routledge Studies in Ancient History, London and New York: Routledge Press.

Lazenby, J. F. (1978): Hannibal's War, England: Aris \& Phillips Ltd., Warminster.

Lazenby, J. F. (1996): The First Punic War, London and New York: Routledge.

Lazenby, J. F. (2004): "Rome and Carthage», en Flower, H. I., The Cambridge Companion to the Roman Republic, Cambridge: Cambridge University Press, pp. 225-241.

Le Bohec, Y. (1996): Histoire militare des guerres puniques. L'art de la guerre, Éditions du Rocher.

Leigh, M. (2004): Comedy and the Rise of Rome, Oxford: Oxford University Press,

Levene, D. S. (2010): Livy on the Hannibalic War, Oxford: Oxford University Press. 
Libourel, J. M. (1973): "A Battle of Uncertain Outcome in the Second Samnite War", The American Journal of Philology 94, n. ${ }^{\circ}$ 1, pp. 71-78.

Liddell HaRT, B. (1926): Scipio Africanus. Greater than Napoleon, London: Greenhill Books.

Loreto, L. (2007): La grande strategia di Roma nell'età della Prima Guerra Punica (ca. 273 - ca. 229 A.C.). L'inizio di un paradosso, Storia politica costituzionale e militare del mondo antico, Napoli: Jovene editore.

McDonnell, M. (2006): Roman manliness. Virtus and the Roman Republic.

Cambridge: Cambridge University Press.

Miltsios, N. (2013): The shaping of narrative in Polybius, Trends in Classics 23, De Gruyter.

OAKLey, S. (2005): A comentary on Livy. Books VI-X. Volume IV: Book X, Oxford: Claredon Press.

Östenberg, I. (2014): "War and Remembrance: Memories of defeat in Ancient Rome», en Alroth, B. y Scheffer, C. (eds.): Attitudes towards the Past in Antiquity: Creating Identities, Stockholm Studies in Classical Archaeology 14, pp. 255-65

Östenberg, I. (2018): "Defeated by the Forest, the Pass, the Wind: Nature as an Enemy of Rome», en Clark, J. H. y Turner, B. (eds.), Brill's Companion to Military Defeat in Ancient Mediterranean Society, Warfare in the Ancient Mediterranean World, Leiden: Brill, pp. 240261.

Palao, J. J. (2016): "La organización de la inteligencia militar y de los "servicios secretos" en el ejército romano", en Martínez, E., Cantera, J. y De Pazzis, M. (eds.): La organización de los ejércitos, Cátedra Extraordinaria Complutense de Historia Militar, Madrid: Ministerio de Defensa, pp. 1:123-157.

Pasco-Pranger, M. (2012): "Naming Cato(s)", The Classical Journal 108, n. ${ }^{\circ}$ 1, pp. 1-3999)5.

Pérez, A. (1999): "Atanagrvm vrbem, qvae capvt eivs popvli erat. El problema de Atanagrvm y la capitalidad Ilergete», Hispania Antiqua, 23, pp. 25-47.

Perley, S. M. (2016): Arcana Imperii: Roman Political Intelligence, Counterintelligence, and Covert Action in the Mid-Republic, Tesis doctoral, Australian National University.

Poccetti, P. (2002): "Continuità e fratture nell'eta della battaglia del Sentino: Note sugli Elogia Scipionici», en Poli, D. (ed.): La battaglia del Sentino. Scontro fra nazioni e incontro in una nazione, Roma: Il Calamo, pp. 681-705. 
Quesada Sanz, F. (2005): «De guerreros a soldados. El ejército de Aníbal como un ejército cartaginés atípico", en Guerra y ejército en el mundo Fenicio-Púnico, pp. 129-161.

Quesada SANZ, F. (2006): "Not so different: individual fighting techniques and small unit tactics of Roman and Iberian armies within the framework of warfare in the Hellenistic Age», Pallas, 70, pp. 245-263.

Quesada Sanz, F. (2015): "Los Escipiones, generales de Roma", en Bendala, M. (ed.): Los Escipiones. Roma conquista Hispania. Catálogo de la Exposición. Museo Arqueológico Regional de la Comunidad de Madrid, febrero-septiembre 2016, Madrid: Comunidad de Madrid, pp. 68-89.

Quesada Sanz, F. (2017): "Los antagonistas en las guerras: Mitos, concepciones de la guerra y efectivos", en Schulten y el descubrimiento de Nvmantia, Madrid: Museo Arqueológico Nacional, pp. 205-225.

Rankov, B. (2011): "A War of Phases: Strategies and Stalemates 264241", en Hoyos, D. (ed.): A Companion to the Punic Wars, Blackwell Companions to the Ancient World, Malden: Blackwell Publishing, pp. 149-166.

Rankov, B. (2015): "Intelligence: Republic", en Le Bohec, Y. (ed.): The Encyclopedia of the Roman Army, I: EAS-POL, Wiley-Blackwell, pp. 541-543.

Rawlings, L. (2007): "Army and Battle During the Conquest of Italy (350264 BC)», en Erdkamp, P. (ed.): A Companion to the Roman Army, The Blackwell Companion to the Ancient World, Malden-Oxford-Victoria: Blackwell Publishing, pp. 45-62.

Rawlings, L. (2011): "The War in Italy, 218-203", en Hoyos, D. (ed.): $A$ Companion to the Punic Wars, Blackwell Companions to the Ancient World, Malden: Blackwell Publishing, pp. 299-319.

Rawson, E. (1971): "The Literary Sources for the Pre-Marian Army", Papers of the British School at Rome, 39, pp. 13-31.

Rosenstein, N. (2004): Rome at War. Farms, Families, and Death in the Middle Republic, Chapel Hill and London: The University of North Carolina Press.

Rossi, A. (2004): "Parallel Lives: Hannibal and Scipio in Livy's Third Decade", Transactions of the American Philological Association, 134, n. ${ }^{\circ}$ 2, pp. 359-381.

Russell, F. (2013): "Finding the Enemy: Military Intelligence», en CAMPBell, B. y Lawrence, A. T.: The Oxford Handbook of Warfare in the Classical World, New York: Oxford University Press, pp. 514-531.

SAGE, M. M. (1979): "The "Elogia" of the Augustan Forum and the "de viris illustribus", Historia: Zeitschrift für Alte Geschichte, 28, n. ${ }^{\circ}$ 2, pp. 192 210. 
SAnctis, G. DE (1916): Storia dei Romani, vol. III, Il pensiero storico 38, Firenze: La Nuova Italia.

Schepens, G. (2013): "Lo sfruttamento militare e politico della memoria e della storia: a propósito del frammento de Sosilo sulla battaglia dell'Ebro", Studi Ellenistici, 27, pp. 385-409.

Scullard, H. H. (1969): A bistory of the Roman Wold 753 to 146 BC, Methuen's history of the Greek and Roman World, London: Methuen \& Co. Ltd.

SHeldon, R. M. (1987): "Hannibal's Spies», International Journal of Intelligence and CounterIntelligence, 1, n. ${ }^{\circ}$ 3, pp. 53-70.

SHeldon, R. M. (2002): "Caesar, Intelligence, and Ancient Britain", International Journal of Intelligence and CounterIntelligence, 15, n. 1, pp. 77-100.

Sheldon, R. M. (2005): Intelligence Activities in Ancient Rome. Trust in the Gods, but Verify, New York and London: Routledge.

Southern, P. (2006): The Roman Army. A Social and Institutional History, Santa Barbara: BC-Clio.

Steinby, C. (2014): Rome versus Carthage: the war at sea, Pen and Sword Maritime, Barnsley: Barnsley.

VACANTI, C. (2012): Guerra per la Sicilia e guerra della Sicilia. Il ruolo delle città siciliane nel primo conflitto romano-punico, Storia politica costituzionale e militare del mondo antico, Napoli: Jovene editore.

Valdés Matías, P. (2017): La logística del ejército romano durante la República Media (264-188 a.C.), Universitat de Barcelona.

Walbank, F. W. (1970): A historical commentary on Polybius I, vol. 1. 3 vols., Oxford: Oxford University Press, 1970.

Walker, D. "Learning from experience: Polybius and the progress of Rome» 67, n. ${ }^{\circ}$ 1, pp. 132-148.

WheELER, E. L. (1988): "Sapiens" and Stratagems: The Neglected Meaning of a "Cognomen"”, Historia: Zeitschrift für Alte Geschichte, 37, n. ${ }^{\circ} 2$, pp. 166-195.

WHEELER, E. L. (1988): Stratagem and the vocabulary of military trickery, 108, Brill.

White, A. (2014): "Hannibal's night time antics: Livy's use of "The Night” in the Third Decade to present military operations, develop moral exempla and examine Rome's past», Degree of Master of Arts in Classics, Classics Department, University of Canterbury.

WolfBerg, A. (2016): "When generals consume intelligence: the problems that arise and how they solve them", Intelligence and National Security, pp. 1-19. 
